\title{
Diferencias de Género y Estudios de Acceso en las Creencias del Alumnado de Grado en Educación Infantil sobre el Desarrollo de la Autonomía en el Ciclo 0-3
}

\author{
Differences in Childhood Education Degree Students' Beliefs \\ about Child Autonomy in the First Cycle (0-3 years) according \\ to Gender and Studies of Origin
}

\author{
Elena Herrán Izagirre \\ Nuria Galende Pérez * \\ Gorka Etxebarria Elordui
}

Universidad del País Vasco / Euskal Herriko Unibertsitatea, España

\begin{abstract}
Este estudio tiene como objetivo profundizar en las creencias y actitudes del alumnado del Grado de Educación Infantil sobre el desarrollo de la autonomía del bebé y niño pequeño (O-3 años). La autonomía temprana se entiende como capacidad preprogramada que impulsa a la cría humana a interactuar con el mundo circundante, tanto físico como humano, de manera progresivamente más activa e independiente. En concreto, el estudio trata de analizar las diferencias en dichas creencias según el género y los estudios de procedencia, en 165 estudiantes universitarios del mencionado Grado. Las respuestas dadas a un cuestionario desarrollado al efecto se mostraron inconsistentes, por lo que se procedió a centrar las puntuaciones. Los resultados muestran diferencias por género coherentes con el carácter feminizado de la profesión y de los estudios. Asimismo, el alumnado proveniente del Grado Superior en Educación Infantil de Formación Profesional tiende a valorar mejor los aspectos prácticos de la profesión que los teóricos o de fundamentación. Esto confirma que los imaginarios de los colectivos que forman la muestra son diferentes según las dos variables elegidas, y plantea un gran reto a la formación de Grado.
\end{abstract}

Palabras clave: Creencia; Autonomía; Título universitario; Educación preescolar; Diferencia de sexo; Enseñanza y formación.

\begin{abstract}
This study aims to go in depth in the beliefs and attitudes of the students of the Degree in Early Childhood Education in relation to the development of the autonomy of babies and toddlers (O-3 years). Early autonomy is understood as a preprogrammed capacity that encourages human breeding to interact with the surrounding world, physical and human, in a progressively more active and independent manner. Specifically, it tries to analyze the differences in these beliefs according to gender and studies of origin in 165 university students of the aforementioned Degree. The answers given to a questionnaire developed for this purpose were inconsistent, so the scores were centered. The results obtained show significant differences by gender coherent with the feminized character of the profession and of the studies. Likewise, students that come from the Higher Degree in Pre-School Education tend to value differentially the practical aspects of the profession more than the theoretical ones. This confirms that the imaginaries of the collectives that form the sample are different according to the two variables chosen, and poses a great challenge to the training in the Degree.
\end{abstract}

Keywords: Belief; Autonomy; Academic degree; Pre-school education; Sex difference; Teaching and training.

*Contacto: nuria.galende@ehu.eus

issn: 1989-0397

www.rinace.net/riee/

https://revistas.uam.es/riee
Recibido: 1 de octubre de 2019

$1^{\text {a }}$ Evaluación: 8 de enero de 2020

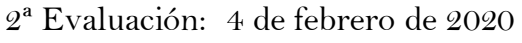

Aceptado: 6 de marzo de 2020 


\section{Introducción}

El ámbito científico de esta área de investigación lleva largo tiempo demostrando la importancia vital de la primera infancia en la construcción sana del psiquismo de las personas (Sánchez-Rodríguez, 2014), así como la prevalencia de dicha construcción a lo largo de la vida, condicionando, entre otras cosas, las futuras interacciones sociales (Raby et al., 2015) así como el conocimiento (Gopnik, 2010). No obstante, a pesar del inmenso volumen de datos que certifica la importancia de invertir en cuidados de calidad en este primer periodo de la vida, la evidencia científica no ha logrado calar en la sociedad, ni, lo que resulta más alarmante, en el sistema educativo en general (Herrán, Orejudo, Martínez de Morentin y Ordeñana, 2014). Esto resulta especialmente preocupante en los niveles educativos superiores (universidad, ciclos formativos), ya que son los estudios mediante los cuales se forma a los y las futuras profesionales que atenderán a bebés y niños pequeños. Los planes de estudios actuales tienden a centrarse en las capacidades cognitivas de la infancia, aplicando objetivos, contenidos, etc., propios de edades ulteriores: el segundo ciclo de Educación Infantil o Primaria. Sin embargo, para que esas capacidades puedan desarrollarse es imprescindible cubrir y responder, previamente, a la necesidad humana más primordial -la construcción del psiquismo-, que, durante toda la vida, pero muy especialmente en la infancia, depende $y$, de hecho, se construye a partir del progresivo dominio y control del propio cuerpo.

Es lo que se conoce como autonomía, definida como la capacidad de una persona de gobernarse a sí misma, de asumir la plena responsabilidad de sus actos. Familias y profesionales de la primera infancia desean ver que los niños se hacen autónomos según esta definición, pero el camino por el que podrán llegar a serlo no siempre está claro, lo que conduce a puntos de vista muy diversos (Falk, 2018a), y más aun teniendo en cuenta el papel que ejercen las creencias de las personas adultas que trabajarán con estos niños, y que no siempre tienen una base científica.

Esa es la razón por la que conocer las creencias del alumnado del Grado de Educación Infantil y del Ciclo Superior de Educación Infantil, en tanto que guías de su futuro comportamiento profesional, es fundamental. Las tendencias de comportamiento educativo se mantienen prácticamente inalterables a pesar de los años de formación universitaria o secundaria- debido al filtro (Lortie, 2002) que incorpora los nuevos conocimientos a los anteriores más arraigados y resistentes, deformándolos y eliminando de ellos las cuestiones disonantes, y las contradicciones paradigmáticas y conceptuales de la propia formación. Es decir, la incorporación de nuevos aprendizajes es limitada.

Todo ello subraya la responsabilidad formativa de la Universidad; es decir, la nuestra. Los cambios son complejos, pero resultan imprescindibles en la CAPV, puntera en el Estado, donde existe una tasa de escolarización temprana que se mantiene alrededor del $52 \%$ desde 2010 -según el EUSTAT (2018) la tasa de matriculación del curso 2016-17 fue del 17,5 \% y $45,7 \%$ sobre el total de 0 y 1 años, respectivamente, y del $93,5 \%$ en las aulas de 2 años. Esta investigación pretende dar con las claves de los ajustes imprescindibles para una Educación Infantil 0-3 años de excelencia. 


\section{Fundamentación teórica}

La autonomía temprana (Falk, 2018a; Kamii, 1982) es un ámbito central de Educación Infantil (E. I.). Es una capacidad preprogramada que impulsa a la cría humana a interactuar con el mundo circundante, físico y humano (Tomasello, 2007; Wallon, 1985), de manera progresivamente más activa e independiente, condicionando por ello los demás ámbitos de desarrollo temprano. Si no hay afecto y respeto en el trato entre el adulto y el niño, puede degenerar fácilmente en heteronomía (Kamii, 1982), “...ser gobernado por algún otro" (p. 4), o en “...las tres grandes trampas de la falsa autonomía: el condicionamiento, una exigencia de precocidad, de la que puede desprenderse una actitud de indiferencia y de abandono, y una actitud de laissez faire" (Falk, 2018a, p. 111). En la primera infancia la calidad de la crianza es clave, ya que apoyar la autonomía ayuda al bebé y niño pequeño a desarrollar estrategias de autocontrol (Tarullo, Obradovic y Gunnar, 2009), además de ser el mejor predictor del desarrollo de las funciones ejecutivas tempranas implicadas en él (Field, 2010; Poulton, Moffitt y Silva, 2015). El autocontrol (Moffitt et al, 2011) se asocia al éxito en la vida adulta (Field, 2010; Poulton et al, 2015) y a diferencia de la inteligencia o el estatus económico, es una variable predictora del mismo (Duckworth, 2011) y además de fácil mejora mediante intervención (Moffitt et. al., 2011).

En relación con la autonomía en E. I., se ha podido establecer cierta diferencia entre dos tipos de creencias: por una parte, creencias profesionales más tradicionales o adultocéntricas, con cierta visión deficitaria o de percepción de limitaciones en lugar de centrarse y desarrollar las potencialidades y capacidades de la primera infancia (Davis y Degotardi, 2015); y, por otra parte, tendencias más progresistas o paidocéntricas, que proponen lo que es mejor y más beneficioso, por adecuado, en cada periodo particular del desarrollo y para cada niño individual (Tonyan, Mamikonian-Zarpas y Chien, 2013). Este último tipo de creencias se ha relacionado con un cuidado más positivo y con la provisión de un entorno más rico y estimulante para los niños, como el de la educación Pikler-Lóczy (David y Appell, 2010; González-Mena, 2004; Herrán, 2013; Herrán et al, 2014), con independencia de otro tipo de variables como el tamaño de grupo (Clarke-Stewart, Vandell, Burchinal, O'Brien y McCartney, 2002). Las creencias más tradicionales parecen ser la razón por la que consolidados resultados de la investigación científica sobre la autonomía temprana no influyen en el conocimiento general ni en la práctica educativa (Pikler, 2018).

Las aproximaciones sobre la enseñanza de los docentes también están relacionadas con sus concepciones de la misma (Trigwell y Prosser, 1996); nociones, ideas previas, representaciones y creencias condicionan su práctica educativa. Así, las diferentes prácticas evaluativas universitarias evidencian diferentes prácticas docentes (Samuelowicz y Bain, 2002) que incluso pueden ser contradictorias (Ribeiro y Flores, 2016), porque los diferentes conceptos de enseñanza, aprendizaje y evaluación asociados influyen diferencialmente en cómo enseña el profesorado y en cómo aprende el alumnado (Brown, 2004; Thompson, 1992). En este sentido, y en el ámbito de las ciencias, tecnología y matemáticas, se ha incrementado la investigación sobre motivaciones, actitudes, expectativas y especialmente, concepciones, de futuros docentes (Brown, Lake y Matters, 2011; Meirik, Meijer, Verloop y Bergen, 2009), con el objetivo de diseñar propuestas metodológicas que favorezcan el cambio de sus ideas previas (Pontes, Poyato y Oliva, 2016). En relación con las representaciones y creencias del alumnado de magisterio, el 
comportamiento docente se apoya en una multiplicidad de factores relacionados con una ideología o percepción de la realidad que asume el-la educador-a, y que se concretan en las múltiples dimensiones de un gran impacto en los estilos de enseñanza, en el ejercicio de la profesión, en la configuración pedagógica del quehacer educativo y en las relaciones interpersonales que se establecen dentro del aula (González-Peiteado y Pino-Yuste, 2014).

Asociada a todo lo anterior, en la formación de los futuros profesionales habría otra cuestión fundamental a tener en cuenta: el filtro intuitivo (Goodman, 1988) con el que se procesan las subsiguientes experiencias educativas y docentes (Lortie, 2002). Este filtro se constituye a partir de las creencias o representaciones sociales de cada cual, en tanto que guías básicas de pensamiento o tamiz que filtrará o no la nueva formación académica. De hecho, Akin (2013) afirma que los alumnos en formación en E. I. utilizan sus creencias propias y arraigadas para evaluar las nuevas ideas, desestimando las que chocan con ellas, tachándolas de teóricas, irrealizables o simplemente incorrectas. Entre ambas -creencias y nuevas informaciones- es imprescindible cierta congruencia (Opfer y Pedder, 2011; Opfer, Pedder, y Lavicza, 2011). De lo contrario, queda muy limitada la adquisición (Goodman, 1988; Raths, 2001) así como el provecho de las subsiguientes experiencias educativas y docentes (Lortie, 2002).

Debido a que estas creencias tienen necesariamente un origen previo al universitario y un arraigo fuerte y profundo, es oportuno hacer referencia a los estilos educativos parentales como modelo explicativo del desarrollo de las creencias sobre el despliegue de la autonomía del niño. Los estilos parentales los conforman metas de desarrollo y estrategias de socialización (Goodnow, 1985) entendidas como tendencias de comportamiento paterno, globales, estables y abiertas, con una gran repercusión y consecuencias evolutivas no solo en la infancia sino en la adolescencia. Se trata de conductas parentales relativas a la disciplina, control, dominio, apoyo, etc. (Baumrind, 1991; Erikson, 1963; Gadeyne, Ghesquière y Onghena, 2004; Rollins y Thomas, 1979). La combinación de estos aspectos ha conformado los denominados estilos educativos parentales: autoritario-recíproco, autoritario-represivo, permisivo-indulgente y permisivo negligente (MacCoby y Martin, 1983). Hoffman (1970) incluye los modelos autoritario, la retirada de afecto, y la inducción, mientras que Kellerhalls y Montandon (1997) proponen los estilos familiares contractualista, estatutario y el maternalista. Todos ellos incluyen, en diferente intensidad, reciprocidad e implicación afectiva o ausencia de ambas; control fuerte o laxo; retirada de afecto o enfado; amenaza o utilización del castigo; imposición o bien dejación o uso de la inducción y oscilan en direcciones opuestas consolidando uno u otro modelo.

Es complejo conocer la influencia de la formación académica universitaria en relación con el ámbito de conocimiento de la autonomía infantil temprana. Entre otras razones, una significativa es la existencia de contradicciones en el propio curriculum del Grado de E. I., asociadas al habitual trasvase de contenidos de ciclos ulteriores a edades tempranas (Loizou y Recchia, 2018). En cualquier caso, el profesor en formación construye su propio perfil de educador informado en función del efecto transformador de las experiencias formativas del Grado en E. I., de cómo cuestionan, replantean y dan alternativa a preconcepciones y creencias anteriores sobre situaciones educativas en las que la autonomía infantil está presente y conforman la tarea del profesional de la educación temprana. Para comprender estos procesos, se elaboró una escala que pretende evaluar las pautas socializadoras y educativas concretas relacionadas con la autonomía y contextualizadas en el primer ciclo de E. I. (O-3 años). Se trata de descubrir si el 
conocimiento adquirido sobre la autonomía infantil temprana y sus condiciones se refleja en las respuestas, en relación a dos variables intervinientes, asociándose a creencias informadas y complejas en lugar de a creencias más tradicionales o simplistas, propias de sus contextos previos y no formativos.

\section{Método}

\subsection{Objetivos}

Este estudio, de tipo comparativo o diferencial, pretende analizar las creencias que conforman los diferentes imaginarios de los colectivos que forman la muestra según dos variables: género y estudios de acceso al Grado en E. I. En concreto, se espera que, al tratarse de una profesión feminizada, haya diferencias en la valoración de las cuestiones asociadas al género. También se espera que el alumnado del Grado en E. I. que proviene del Grado Superior en E. I. de Formación Profesional evidencie su propio filtro, más práctico que el de la formación previa de Bachillerato.

\subsection{Participantes}

Son 165 participantes, estudiantes universitarios de Grado en E. I., 86\% mujeres, con edades comprendidas entre los 19 y los 66 años. El 54\% de la muestra tiene la edad habitual para los estudios que cursan, 19-20 años; el 33\% tiene entre 21 y 24 años y el 13\% restante tiene edades superiores a los 24 años. La edad está asociada a la vía de acceso a los estudios de grado: Bachillerato, Formación Profesional y Acceso a la Universidad para mayores de 25 años. Los datos se han recogido en dos centros distintos, uno de titularidad pública y el otro privada, de la Comunidad Autónoma del País Vasco. Cabe señalar que 41 de los 165 participantes tienen cursado el Grado Superior en E. I. de Formación Profesional. El muestreo ha sido de conveniencia, seleccionando los centros y grupos/aula que ofrecían mejores garantías y condiciones de aplicabilidad, primando la validez ecológica a la representatividad estrictamente aleatoria. Como resultado de estos criterios de selección, el tamaño de la muestra final se considera suficiente para garantizar un buen nivel de "poder" en los contrastes estadísticos aplicados. Las pruebas de significación estadística se acompañan de los coeficientes de tamaño del efecto con el fin de que pueda apreciarse también la cuantía absoluta de las diferencias.

\subsection{Instrumento}

Las pautas socializadoras y educativas relacionadas con la autonomía y contextualizadas en el primer ciclo ( $0-3$ años) de E. I. a analizar se han rescatado del cuestionario CUIDANDO 0-2 (Herrán et al., 2014) aplicado a profesionales en ejercicio, adaptándolo al profesorado en formación. Así, se identifican las mismas siete dimensiones o facetas relacionadas con la autonomía infantil con sus correspondientes ítems: concepto de niño (ej.: la educación temprana es buena para la salud del niño de 0-3 años), rol de la educadora (ej.: es imprescindible la intervención del adulto para solucionar los conflictos entre niños), actividad diaria (ej.: a la hora de la siesta todos los niños deberían dormir), sentimientos asociados a la actividad (ej.: creo que mis abrazos, besos y caricias son imprescindibles para el bienestar del niño), interacción con los niños (ej.: disfrutaría del momento del cambio de pañal a los niños/as de 0-3 años.), relación con la pareja educativa y evaluación (ej.: es necesario negociar y compartir los objetivos del primer ciclo (0-3 años) con la pareja 
pedagógica), evaluación, innovación y mejora (ej.: compartiré a diario las observaciones con mis compañeras).

Así, se han diseñado 35 ítems con los que se pretende indagar sobre las creencias relativas a las pautas educativas y de crianza autónomas del alumnado del grado en tanto que futuros profesionales de la primera infancia. Todos los ítems se ordenarían según las dos modalidades de intervención: directa e indirecta. La primera reúne exclusivamente los ítems relacionados con el hacer individual en vivo y en directo a los bebés y niños pequeños, mientras que la segunda incluiría todo lo demás: contexto educativo, opiniones, valoraciones o perfil educador. En el proceso de construcción del instrumento se contó con el criterio de 20 profesionales expertos, que aportaron validez de contenido a la elaboración y selección de los ítems.

\subsection{Procedimiento de recogida y análisis de datos}

En primer lugar, se realizaron los análisis descriptivos básicos, encontrando coeficientes de asimetría y curtosis que mostraron distribuciones muy alejadas de la normalidad en los ítems 7, 17, 19, 26, 31, 33, 34. Estos ítems se retiraron de los análisis posteriores para evitar sesgos importantes en la estimación de los coeficientes.

Los primeros análisis descriptivos y exploratorios mostraron con claridad que el instrumento no medía de manera consistente las dimensiones postuladas. Se exploraron diferentes elementos que explicaran estos resultados, estableciéndose como posibilidad la presencia de sesgos importantes como la aquiescencia, deseabilidad social, radicalidad en las respuestas (Morales, 2000).

Como estrategia para resolver o paliar este problema, se procedió al centrado de las respuestas, utilizando la media de cada sujeto en cada ítem como norma individual (Schwartz, 2003). Con ello, no se trabaja con las puntuaciones brutas, sino con las relativas a la media de cada uno de los sujetos. La puntuación en el ítem refleja, por tanto, si dicho ítem se encuentra entre los más o menos valorados por los participantes, en lugar de su valoración en términos absolutos.

Dado que la orientación del ítem ha mostrado un comportamiento diferencial en numerosos estudios previos (Solís, 2015), se analizan por separado los ítems orientados positivamente o favorables a la autonomía temprana y los orientados negativamente o favorables a la heteronomía. El primer grupo lo conforman 15 ítems -6 directos y 9 indirectos- relativos a contenidos trabajados en el Grado en E. I., o creencias informadas sobre la autonomía temprana, fruto de la investigación en primera infancia. El segundo, formado por 13 ítems -6 directos y 7 indirectos- agrupa contenidos y creencias ajenas a los contenidos del Grado y por ello, creencias más simplistas que trae el alumnado, asociadas a la heteronomía temprana.

Para constatar posibles diferencias según las variables criterio mencionadas, se utilizó el Análisis de la Varianza, que identificó la existencia o no de diferencias estadísticamente significativas entre los grupos. Para estimar el tamaño del efecto, se utilizó el coeficiente eta. Los cuadros se presentan ordenadas por tamaño del efecto de manera que se puede identificar con claridad en qué ítems aparecen diferencias de mayor tamaño. 


\section{Resultados y conclusiones}

El cuadro 1 presenta las diferencias encontradas según el género en los ítems orientados hacia el desarrollo de la autonomía.

Cuadro 1. Puntuaciones centradas de los ítems orientados hacia la autonomía según el género

\begin{tabular}{|c|c|c|c|c|c|c|}
\hline & $\begin{array}{r}\text { MED } \\
\text { MUJER }\end{array}$ & HOMBRE & TOTAL & $\mathbf{F}$ & SIG. & $\mathbf{E T A}^{2}$ \\
\hline $\begin{array}{l}\text { 24. Dejaría a mis hijos/as en manos de mis propios } \\
\text { compañeros/as de Grado /Ciclo. }\end{array}$ &,- 58 &, 14 &,- 48 & 8,297 & ,005 & ,050 \\
\hline $\begin{array}{l}\text { 06. En el período de adaptación haré todo lo que esté en } \\
\text { mis manos para que los niños/as no lloren cuando se } \\
\text { separen de sus padres. }\end{array}$ & ,04 &,- 59 &,- 04 & 5,993 & ,015 & ,036 \\
\hline $\begin{array}{l}\text { 25. Para ser un buen profesional es más importante ser reflexivo } \\
\text { que práctico.* }\end{array}$ & $-1,02$ &,- 50 &,- 95 & 5,621 & ,019 & ,034 \\
\hline $\begin{array}{l}\text { 28. Es importante planificar el día a día de la escuela en } \\
\text { torno a los cuidados (cambio de pañal, higiene, } \\
\text { alimentación...). }\end{array}$ &, 81 &, 50 & ,77 & 3,619 & ,059 & ,022 \\
\hline $\begin{array}{l}\text { 22. Compartiré a diario mis observaciones con mis } \\
\text { compañeras/os. }\end{array}$ & s, 13 & ,36 &, 16 & 1,405 & ,238 & ,009 \\
\hline $\begin{array}{l}\text { 18. Me gustará tratar con los/las padres/familias de los } \\
\text { niños y niñas. }\end{array}$ & s, 67 &, 50 & ,65 & 1,075 &, 301 & ,007 \\
\hline $\begin{array}{l}\text { 32. En el aula de dos años la tutora debe cambiar pañales } \\
\text { al igual que la auxiliar. }\end{array}$ & s 49 & ,68 & ,52 & 1,013 & ,316 & ,006 \\
\hline $\begin{array}{l}\text { 11. Disfrutaría del momento del cambio de pañal a los } \\
\text { niños/as de 0-3 años. }\end{array}$ & 35 &,- 59 &,- 39 & 1,005 & ,318 & ,006 \\
\hline $\begin{array}{l}\text { 20. Me preocupa si sabré gestionar adecuadamente en el } \\
\text { día a día la acogida y despedida de los niños. }\end{array}$ &,- 57 &,- 77 &,- 60 & ,623 & , 431 & ,004 \\
\hline $\begin{array}{l}\text { 35. Es necesario negociar y compartir los objetivos del } \\
\text { primer ciclo (0-3 años) con la pareja pedagógica. }\end{array}$ & , 83 & ,91 & 84 & ,385 & ,536 & ,002 \\
\hline 21. Soy capaz de realizar mi trabajo en el ciclo 0-3 años. & ,34 & ,42 & ,35 &, 196 & ,659 & ,001 \\
\hline $\begin{array}{l}\text { 13. Antes de quitarle los mocos a un niño/a le avisaría de } \\
\text { lo que le voy a hacer. }\end{array}$ & e, 73 & ,66 & ,72 & , 192 & 662 & ,001 \\
\hline $\begin{array}{l}\text { 10. En las comidas dejaría que los propios niños eligiesen } \\
\text { la cantidad que van a comer. }\end{array}$ & $-1,39$ & $-1,50$ & $-1,40$ & , 173 & ,678 & ,001 \\
\hline $\begin{array}{l}\text { 04. Dejar al niño que adopte la postura que quiera en el } \\
\text { cambio de pañal refuerza su seguridad. }\end{array}$ &,- 64 &,- 68 &,- 65 & ,019 & ,891 & ,000 \\
\hline $\begin{array}{l}\text { 29. Cuando un niño que quita a otro un juguete tenemos } \\
\text { que recordarle que no lo puede hacer mientras esté } \\
\text { jugando con él. }\end{array}$ & ,, 51 &, 50 & ,51 & & 969 & ,000 \\
\hline
\end{tabular}

Mujeres ( $\mathrm{n}=139)$ Hombres $(\mathrm{n}=22)$ Total $(\mathrm{n}=161)$

* Los ítems indirectos van en cursiva en todas los cuadros para facilitar la lectura.

Fuente: Elaboración propia.

Encontramos tres ítems con diferencias estadísticamente significativas $(\mathrm{p}<, 05)$, dos directos -24, 06- y uno indirecto -25-. En el ítem 24-dejar a los propios hijos con los colegas- las mujeres otorgan una valoración muy negativa $(-, 58)$ mientras que los hombres otorgan una valoración media $(, 14)$. La valoración del ítem 06 -preocupación por el periodo de adaptación- es media en el caso de las mujeres (,04) y negativa en el caso de los hombres (,59). En el ítem indirecto -25-, la valoración sobre ser reflexivo frente a práctico es negativa en ambos colectivos, pero extremadamente negativa entre las mujeres $(-1,02)$ frente a los hombres $(-, 50)$.

Los resultados señalarían, pues, algunas diferencias asociadas al género. Así, las mujeres mostrarían una evidente desconfianza hacia sus compañeros, lo que podría ser coherente con el carácter marcadamente feminizado de la profesión (Anliak y Beyazkurk, 2008), además de relacionarse con el estilo millennial (Clark y Byrnes, 2015), que se siente por 
encima de la media en diferentes aspectos. También éstas se preocuparían poco en la adaptación, aunque bastante más que los hombres, y se decantarían diferencialmente por la practicidad frente a la reflexión (Avgitidou, Pnevmatikos, y Likomitrou, 2013; Vartuli y Rohs, 2009).

El cuadro 2 presenta las diferencias encontradas según el género en los ítems orientados hacia el desarrollo de la heteronomía.

Cuadro 2. Puntuaciones centradas de los ítems orientados hacia la heteronomía según género

\begin{tabular}{|c|c|c|c|c|c|c|}
\hline & \multicolumn{3}{|c|}{ MEDIA } & \multirow[b]{2}{*}{$\mathbf{F}$} & \multirow[b]{2}{*}{ SIG. } & \multirow[b]{2}{*}{ ETA } \\
\hline & MUJER & HOMBRE & TOTAL & & & \\
\hline $\begin{array}{l}\text { O2. Me tranquilizaría establecer normas estrictas en el } \\
\text { aula para evitar el caos. }\end{array}$ &,- 97 &,- 45 &,- 90 & 5,139 &, 025 & ,031 \\
\hline $\begin{array}{l}\text { 12. A la hora de comer me quedaría realmente } \\
\text { tranquila/o cuando viese que el/la niño/a ha terminado } \\
\text { lo que tenía en el plato. }\end{array}$ &, 45 & ,OO & ,39 & 4,656 & ,032 & ,028 \\
\hline $\begin{array}{l}\text { 14. En mi opinión, para que un niño/a aprenda a } \\
\text { controlar los esfínteres, es adecuado ponerle a la misma } \\
\text { hora en el orinal }\end{array}$ & ,20 &,- 27 & , 13 & 4,583 & ,034 & ,028 \\
\hline $\begin{array}{l}\text { 30. Aunque la adaptación sea mala el niño pueden } \\
\text { construir un buen vínculo sano con la educadora }\end{array}$ &, 61 &, 14 &, 54 & 3,601 & ,060 & ,022 \\
\hline $\begin{array}{l}\text { 08. Si sientan al niño/a antes de estar preparado, yo no lo } \\
\text { sentaría en el aula. }\end{array}$ &,- 22 &, 17 &,- 17 & 1,800 & , 182 & ,011 \\
\hline $\begin{array}{l}\text { O1. Es básico que el niño/a se relacione, desde bebé, con } \\
\text { la mayor cantidad de educadores/as posible desde el } \\
\text { inicio de su escolarización. }\end{array}$ & $-1,20$ &,- 93 & $-1,17$ & 1,165 &, 282 & ,007 \\
\hline $\begin{array}{l}\text { 23. En el prácticum se aprende más sobre la educación de } \\
\text { los niños y las niñas de esta edad que en todo el resto del } \\
\text { grado/ciclo. }\end{array}$ &, 74 &, 59 &, 72 & ,437 &, 510 & ,003 \\
\hline $\begin{array}{l}\text { 03. Es más importante respetar el movimiento libre del } \\
\text { niño en los momentos de actividad autónoma que durante } \\
\text { los cuidados (cambio de pañal, higiene, alimentación...). }\end{array}$ & ,03 &, 18 & ,05 & ,278 & ,599 & ,002 \\
\hline $\begin{array}{l}\text { 15. A la hora de la siesta todos los niños/as deberían } \\
\text { dormir. }\end{array}$ &,- 63 &,- 54 &,- 61 &, 102 & ,750 & ,001 \\
\hline $\begin{array}{l}\text { 27. Debemos mostrar nuestro enfado cuando los } \\
\text { niños/as actúan mal. }\end{array}$ &,- 02 & ,05 &,- 01 & ,085 &, 771 &, 001 \\
\hline $\begin{array}{l}\text { o9. Es necesaria la intervención de la educadora para } \\
\text { resolver los conflictos infantiles. }\end{array}$ & ,08 & ,13 & ,09 &, 055 & ,815 & ,000 \\
\hline $\begin{array}{l}\text { 05. La escolarización temprana (0-3) es buena para la } \\
\text { salud de los niños/as. }\end{array}$ & ,06 & ,04 & ,06 & ,005 & ,944 & ,OOO \\
\hline $\begin{array}{l}\text { 16. Considero que mis abrazos, besos y caricias son } \\
\text { imprescindibles para el bienestar del niño. }\end{array}$ &, 87 & ,86 & ,87 & ,003 & ,959 & ,000 \\
\hline
\end{tabular}

Entre los ítems orientados hacia la heteronomía, en relación a la variable género (cuadro 2), encontramos tres ítems directos -02, 12 y 14-, con diferencias estadísticamente significativas $(\mathrm{p}<, 05)$. La valoración del ítem 02 -normas estrictas para evitar el caos- es extremadamente negativa entre las mujeres $(-, 97)$ mientras que entre los hombres $(-, 45)$ es bastante negativa. En el ítem 12 las mujeres valoran positivamente que el niño acabe el plato $(45)$ mientras que los hombres tienen una posición media al respecto (,o0). En el ítem 14 se mantiene esta tendencia: las mujeres valoran de manera moderadamente positiva que se coloque al niño a la misma hora en el orinal para aprender a controlar esfínteres (,20), mientras que los hombres valoran de manera moderadamente negativa esta cuestión $(-, 27)$. 
Las valoraciones de estos tres ítems tienen que ver con la norma y el aprendizaje autoritario de hábitos. Las mujeres serían más permisivas que los hombres con el caos del aula, pero más estrictas en lo relativo a terminar lo servido en el plato o el condicionamiento para el control de esfínteres. Evidentemente, son cuestiones relacionadas con el género. Aunque todo apunta a que, al ser tareas normalmente desarrolladas por la madre en el entorno familiar, habría que indagar si se trata de una cuestión atávica femenina asociada a la ancestral idea de comida-amor (Hamburg, Finkenauer y Schuengel, 2014) o a la exigencia de la limpieza precoz (Falk y Vincze, 2018), y aprendida de esos estilos educativos parentales por el alumnado femenino.

En resumen, los resultados asociados al género señalan algunas diferencias relevantes. Así, los ítems orientados hacia la autonomía dejan translucir que las mujeres mostrarían en conjunto cierta rigidez y exigencia autoritaria (Hoffman, 1970; MacCoby y Martin, 1983) en forma de evidente desconfianza profesional para con sus propios compañeros, escasa preocupación por la inevitable adaptación -aunque bastante más que los hombres en los que a la vista de los demás ítems este hecho podría deberse a dejadez o impotencia (Falk, 2018a)- y nuevamente de practicidad frente a reflexión; cuestiones todas ellas coherentes con el carácter marcadamente feminizado de la profesión, además de con la superioridad del estilo millennial y a la confusión entre realidad y práctica, anteriormente citados. Por su parte, las valoraciones de los tres ítems orientados a la heteronomía seguirían teniendo que ver con la norma autoritaria. Mantienen una diferencia similar por géneros, aunque el primero se decanta en dirección contraria a los otros dos. Así, las mujeres serían más permisivas que los hombres con el caos del aula -inevitable e inherente a la actividad de juego temprano- pero diferencialmente reticentes a la gestión autónoma del hambre y la saciedad (Vincze, 2018) o proclives a promover el control de esfínteres mediante condicionamiento (Falk y Vincze, 2018). Convendría indagar si se trata de una cuestión atávica femenina asociada a la ancestral idea de comida-amor (Hamburg et al. 2014) o a la exigencia de la limpieza precoz, y aprendida de los estilos educativos parentales (Hoffman, 1970; MacCoby y Martin, 1983) por el alumnado femenino, al ser tareas normalmente desarrolladas por la madre en el entorno familiar.

En cuanto a las posibles diferencias según formación previa (Bachillerato vs. FP), el cuadro 3 presenta las encontradas en los ítems orientados hacia el desarrollo de la autonomía.

Como puede observarse (cuadro 3), seis ítems presentan diferencias estadísticamente significativas $(\mathrm{p}<, 05)$ entre el alumnado procedente de Bachiller y el procedente de FP, de los que tres serían directos -11, 06, 29- y otros tres, indirectos -21, 20, 25-.

Entre los primeros, en el 11 - disfrute del cambio de pañal- el alumnado procedente de FP muestra una valoración media y el alumnado procedente de Bachillerato muestra una valoración claramente negativa. Aunque las valoraciones del cambio de pañal son bajas en ambos grupos, la diferencia entre ellas puede deberse a que se trata de un ámbito curricular de la FP y no del grado. Con el ítem 06 -sobre el proceso de adaptación- ocurre lo contrario: los alumnos de Bachillerato otorgan una puntuación media, mientras que los de FP otorgan una valoración claramente negativa (,05 frente a -,41). El ítem 29 se refiere a la gestión de las normas asociadas a la socialización de los juguetes y recibe una valoración positiva en ambos colectivos, pero significativamente superior en el alumnado de Bachiller (,61 frente a ,32 del de FP). De los tres ítems indirectos, en el ítem 21, referido a la propia capacidad para realizar el trabajo, el alumnado de Bachiller otorga una valoración menor 
$(, 25)$ que el de FP (,59), confirmando que estos últimos se sienten más capaces de realizar su trabajo en el ciclo 0-3 años. La valoración del ítem 20 apunta a que a ninguno de los grupos le preocupa la gestión de las transiciones diarias, pero diferencialmente menos al alumnado de FP (-,92) que a los de Bachiller (-,45). Finalmente, la valoración sobre ser reflexivo frente a práctico -ítem 25- es muy baja en ambos colectivos, pero bastante más baja entre el alumnado de FP $(-1,23)$ frente a los de Bachiller $(-, 80)$.

Cuadro 3. Puntuaciones centradas de los ítems orientados hacia la autonomía según formación previa

\begin{tabular}{|c|c|c|c|c|c|c|}
\hline & \multicolumn{3}{|c|}{ MEDIA } & \multirow[b]{2}{*}{ F } & \multirow[b]{2}{*}{ SIG. } & \multirow[b]{2}{*}{ ETA $^{2}$} \\
\hline & BACHILLER & $\mathbf{F P}$ & TOTAL & & & \\
\hline $\begin{array}{l}\text { 11. Disfrutaría del momento del cambio de pañal a los } \\
\text { niños/as de 0-3 años. }\end{array}$ &,- 58 & ,08 &,- 40 & 13,205 & 5,000 & ,082 \\
\hline 21. Soy capaz de realizar mi trabajo en el ciclo O-3 años. &, 25 &, 59 & ,34 & 7,608 & ,007 & ,050 \\
\hline $\begin{array}{l}\text { 20. Me preocupa si sabré gestionar adecuadamente en el día a } \\
\text { dia la acogida y despedida de los niños. }\end{array}$ &,- 45 &,- 92 &,- 58 & 5,904 & ,016 & ,039 \\
\hline $\begin{array}{l}\text { 25. Para ser un buen profesional es más importante ser reflexivo } \\
\text { que práctico. }\end{array}$ &,- 80 & $-1,23$ &,- 92 & 5,832 & ,017 & ,038 \\
\hline $\begin{array}{l}\text { 06. En el período de adaptación haré todo lo que esté en } \\
\text { mis manos para que los niños/as no lloren cuando se } \\
\text { separen de sus padres. }\end{array}$ & ,05 &,- 41 &,- 08 & 1 & ,029 & ,032 \\
\hline $\begin{array}{l}\text { 29. Cuando un niño quita a otro un juguete tenemos que } \\
\text { recordarle que no lo puede hacer mientras esté jugando con } \\
\text { él. }\end{array}$ & ,61 & ,32 & ,53 & 0 & ,047 & ,027 \\
\hline $\begin{array}{l}\text { pte la postura que quiera en el } \\
\text { u seguridad. }\end{array}$ &,- 82 &,- 43 &,- 71 & 3,560 & ,061 &, 024 \\
\hline $\begin{array}{l}\text { a que los propios niños eligiesen } \\
\text { ner. }\end{array}$ & $-1,51$ & $-1,26$ & $-1,44$ & 1,547 & ,216 & ,010 \\
\hline $\begin{array}{l}\text { 28. Es importante planificar el día a día de la escuela en } \\
\text { torno a los cuidados (cambio de pañal, higiene, } \\
\text { alimentación...). }\end{array}$ & ,81 & ,71 & ,79 & 580 & ,447 & ,004 \\
\hline $\begin{array}{l}\text { 13. Antes de quitarle los mocos a un niño/a le avisaría de } \\
\text { lo que le voy a hacer }\end{array}$ &, 75 &, 71 &, 74 &, 137 & ,711 & ,001 \\
\hline $\begin{array}{l}\text { 24. Dejaría a mis hijos/as en manos de mis propios } \\
\text { compañeros/as de Grado/Ciclo. }\end{array}$ &,- 48 &,- 41 &,- 46 & ,129 & ,721 & ,001 \\
\hline $\begin{array}{l}\text { 35. Es necesario negociar y compartir los objetivos del } \\
\text { primer ciclo (0-3 años) con la pareja pedagógica. }\end{array}$ & ,82 & ,86 & ,83 &, 121 &, 728 & ,001 \\
\hline $\begin{array}{l}\text { 32. En el aula de dos años la tutora debe cambiar pañales } \\
\text { al igual que la auxiliar. }\end{array}$ &, 52 &, 57 &, 53 &, 104 &, 748 & ,001 \\
\hline $\begin{array}{l}\text { 22. Compartiré a diario mis observaciones con mis } \\
\text { compañeras/os. }\end{array}$ &, 19 & , 18 &, 19 & ,011 & ,918 & ,000 \\
\hline $\begin{array}{l}\text { 18. Me gustará tratar con los/las padres/familias de los } \\
\text { niños y niñas. }\end{array}$ & ,63 & ,64 & ,63 & ,008 & ,930 & , 000 \\
\hline
\end{tabular}
* Bachiller $(\mathrm{n}=108) ; \mathrm{FP}(\mathrm{n}=41)$; Total $(\mathrm{n}=149)$.

Fuente: Elaboración propia.

Estos resultados evidenciarían cierta fractura entre ambos grupos. Así, el alumnado de FP, a pesar de la formación universitaria compartida con los alumnos provenientes de Bachiller, presentaría un filtro intuitivo (Goodman, 1988) que le haría más resistente a responder a las dificultades de la adaptación o a la socialización de los juguetes, y más proclive a disfrutar más del cambio de pañal -aunque poco-, a sentirse capaces de desarrollar su tarea, a despreocuparse de las entradas y salidas y a valorar la practicidad frente a la reflexión, todo lo cual cuestionaría el objetivo formativo del propio Grado en E. I. Además, esta tendencia podría asociarse a la autosuficiencia del estilo millennial (Clark y Byrnes, 2015), y también a la habitual confusión entre realidad y práctica, que en las creencias simplistas se interpretan como sinónimos, mientras que las más progresistas comparten que un buen análisis y reflexión de la realidad, que incorpora elementos 
teóricos relevantes, se considera lo único que puede guiar una práctica realmente transformadora (Avgitidou et al, 2013; Vartuli y Rohs, 2009).

Cuadro 4. Puntuaciones centradas de los ítems orientados hacia la heteronomía según formación previa

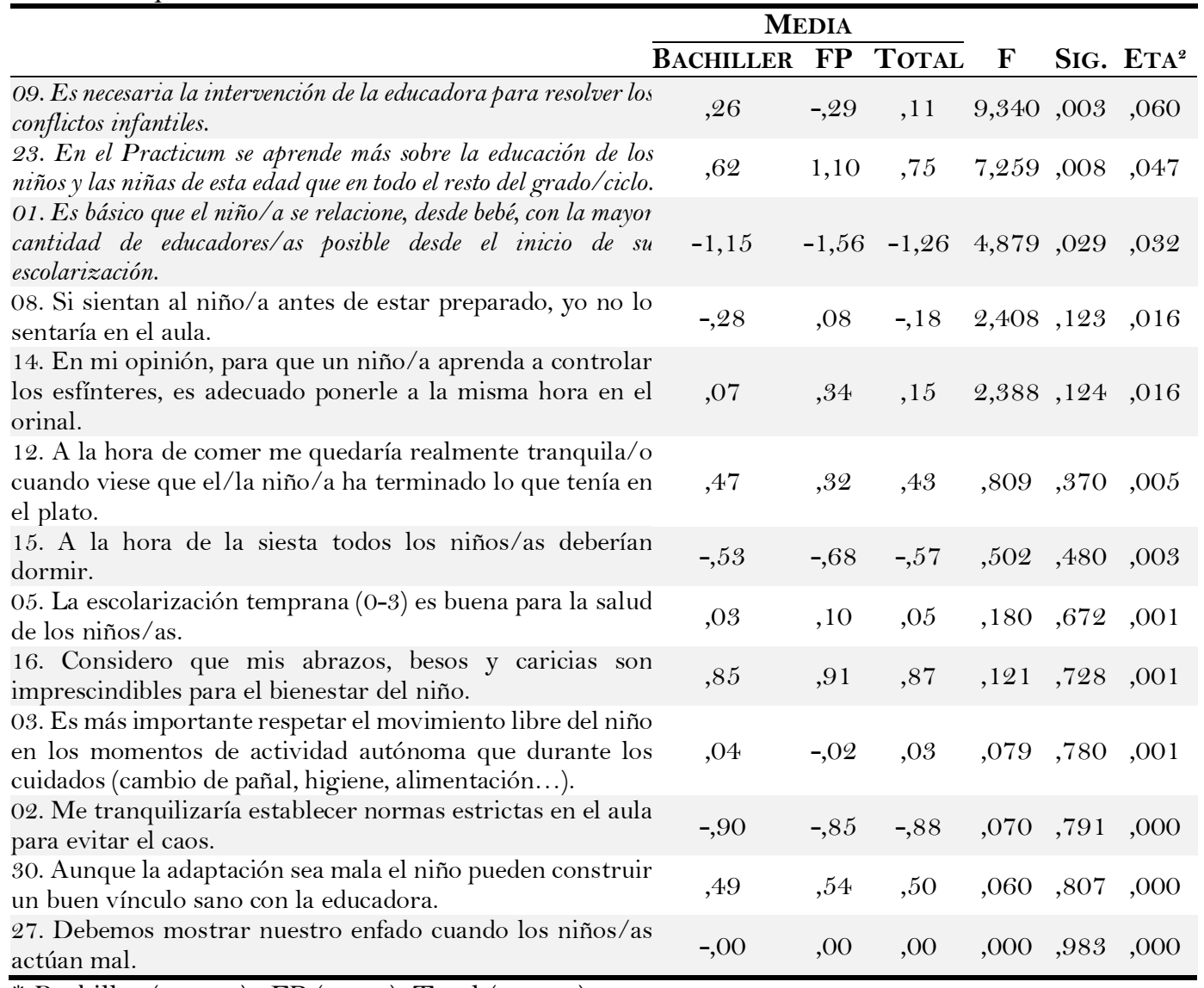

* Bachiller ( $n=108)$; FP $(n=41)$; Total $(n=149)$.

Fuente: Elaboración propia.

Recordemos que los ítems del cuadro 4 se denominan orientados hacia la heteronomía, puesto que las conductas que reflejan serían contrarias al desarrollo de la autonomía. En cuanto a su relación con la variable formación previa, encontramos tres ítems indirectos 09, 23, 01- con diferencias estadísticamente significativas $(\mathrm{p}<, 05)$.

En el ítem 09 la valoración es media-baja para el alumnado de Bachillerato (,26), y bastante más baja para el de FP $(-, 29)$. Es decir, mientras que para el alumnado de Bachiller es adecuado que la educadora intervenga en los conflictos, para el alumnado de FP, no, lo que invierte el sentido del ítem, resultando favorable a la autonomía. El ítem 23 aprendizaje en el prácticum vs. Grado- presenta una valoración positiva media-alta en el caso de los alumnos de Bachiller $(, 62)$ y muy alta $(1,10)$ para el alumnado de FP, lo que supone que ambos grupos valoran más la formación del practicum que la del resto del Grado, pero esta tendencia es extremadamente alta en el caso de los estudiantes que proceden de FP. Finalmente, en el ítem 01 -que bebés y niños pequeños se relacionen con la menor cantidad de educadores posible en el tiempo de la escolarización temprana- se va a producir una inversión, ya que la valoración en ambos grupos es negativa, incluso muy 
negativa para el de FP (-1,56), lo que vuelve a transformar el sentido del ítem en autónomo.

Los resultados informan de que los estudiantes con formación previa en FP invierten el sentido de dos de los ítems, transformándolos en favorables a la autonomía: tienen bastante más claro que los de Bachiller que en la mayoría de los conflictos tempranos no es necesaria la intervención adulta (Tardos y Vasseur-Paumelle, 2018). También saben mejor que las relaciones vinculares en la educación en colectividad deben ser con pocas personas adultas (Falk 2013, 2018b). Nuevamente, la practicidad se impone a la reflexión, aunque diferencialmente, ya que la valoración del alumnado de FP mucho más alta que la de Bachiller (Avgitidou et al., 2013; Vartuli y Rohs, 2009).

En definitiva, los resultados asociados a los estudios de acceso al Grado informan de que entre ambas muestras habría una fractura asociada al filtro intuitivo (Goodman, 1988), que transluce normatividad, sentido del deber o incluso cierto fatalismo (Kellerhalls y Montandon, 1997). Así, el alumnado de FP, a pesar de la formación universitaria compartida con los alumnos provenientes de Bachiller, sería más resistente a responder a las dificultades de la adaptación, a la gestión de la acogida, o a la socialización autónoma de los juguetes, cuestiones inevitables por inherentes a la escolarización temprana, y más proclives a sentirse capaces de hacer su tarea y a vindicar la practicidad frente a la reflexión, cuestionando por todo ello el objetivo formativo del propio Grado en E. I. Esta tendencia, además de asociarse a la autosuficiencia del estilo millennial (Clark y Byrnes, 2015) -se sentirían capaces de hacer algo de lo que se desentienden- podría deberse a la confusión entre realidad y práctica, que en las creencias simplistas se interpretan como sinónimos, mientras que las más progresistas comparten que un buen análisis y reflexión de la realidad, que incorpora elementos teóricos relevantes, se considera lo único que puede guiar una práctica realmente transformadora (Avgitidou et al, 2013; Vartuli y Rohs, 2009). Además, la diferencia en la valoración del cambio de pañal entre ambos colectivos -poco favorable para la FP y bastante desfavorable para Bachiller- evidencia que es un ámbito curricular en la FP y no en el Grado. Los ítems heterónomos informarían de cierta congruencia entre el filtro y la nueva información (Opfer y Pedder, 2011; Opfer et al, 2011): el alumnado proveniente de FP tendría bastante más claro que el de Bachiller que en la mayoría de los conflictos tempranos no sería necesaria la intervención adulta (Tardos y Vasseur-Paumelle, 2018) y también que las relaciones vinculares seguras y estrechas en educación temprana deberían ser con pocas personas adultas (Falk 2013, 2018b).

En síntesis, este estudio diferencial confirma que los imaginarios de los colectivos que forman la muestra son diferentes según las dos variables elegidas: género y estudios de acceso al Grado de Maestro E. I. Los ítems que presentan diferencias significativas son 15 , de los que dos se repiten en dos de las variables -6 y 25- y se refieren a diferentes cuestiones normativas: al compromiso con la tarea educativa temprana y a la dicotomía teoría-práctica.

Además, el alumnado con la formación actual tendría importantes dificultades para deshacer su filtro intuitivo sobre la educación temprana, mayormente normativo y autoritario, y para tomar conciencia de la relevancia de fomentar adecuadamente la autonomía temprana en su tarea diaria, además de aprender a hacerlo en la práctica. Esto nos lleva a plantear un gran reto a la formación de Grado, ya que además de cuestionarse sus contenidos específicos y eliminar los de niveles educativos ulteriores, debe acceder a 
las creencias que conforman los diferentes filtros intuitivos y deshacerlos para poder ofrecer al alumnado una formación realmente informada, veraz y actualizada.

\section{Referencias}

Akin, Z. (2013). Examining the beliefs of Turkish preservice Early Childhood teachers regarding early childhood curriculum. Journal of Research in Childhood Education, 27, 302-318. https://doi.org/10.1080/02568543.2013.796331

Anliak S. y Beyazkurk D. S. (2008). Career perspectives of male students in early childhood education. Educational Studies, 34(4), 309-317.

https://doi.org/10.1080/03055690802034518

Avgitidou, S., Pnevmatikos, D. y Likomitrou, S. (2013). Preservice teachers' beliefs about childhood: challenges for a participatory early childhood education? Journal of Early Childhood Teacher Education, 34(4), 390-404. https://doi.org/10.1080/10901027.2013.845633

Baumrind, D. (1991). Parenting styles and adolescent development. En R. M. Lerner, A. C. Petersen y J. Brooks-Gunn (Eds.), Encyclopedia of adolescence, vol. 2 (pp. 746-758). Garland Publishing.

Brown, G. (2004). Teachers' conceptions of assessment: Implications for policy and professional development. Assessment in Education: Principles, Policy E Practice, 11(3), 301-318. https://doi.org/10.1080/0969594042000304609

Brown, G. T., Lake, R. y Matters, G. (2011). Queensland teachers' conceptions of assessment: The impact of policy priorities on teacher attitudes. Teaching and Teacher Education: An International Journal of Research and Studies, 27(1), 210-220. https://doi.org/10.1016/j.tate.2010.08.003

Clark, S. y Byrnes, D. (2015). What Millennial Preservice Teachers Want to Learn in Their Training. Journal of Early Childhood Teacher Education, 36(4), 379-395. https://doi.org/10.1080/10901027.2015.1100148

Clarke-Stewart, K. A., Vandell, D. L., Burchinal, M., O'Brien, M. y McCartney, K. (2002). Do regulable features of child-care homes affect children's development? Early Childhood Research Quarterly, 17, 52-86. https://doi.org/10.1016/S0885-2006(02)00133-3.

David, M. y Appell, G. (2010). Lóczy. Una insólita atención personal. Octaedro.

Davis, B. y Degotardi, S. (2015). Educators' Understandings of, and Support for, Infant Peer Relationships in Early Childhood Settings. Journal of Early Childhood Research, 13(1), 64-78. https://doi.org/1476718X14538600

Duckworth, A. L. (2011). The significance of self-control. Proceedings of the National Academy of Sciences, 108(7), 2639-2640. https://doi.org/10.1073/pnas.1019725108

Erikson, E. H. (1963). Infancia y Sociedad. Ediciones Horme.

Falk, J. (2013). Si tocamos el cuerpo del bebé. En J. Falk (Ed.), Bañando al bebé. El arte del cuidado (pp. 7-16). Asociación Pikler-Lóczy de Hungría.

Falk, J. (2018a). Los fundamentos de la verdadera autonomía. En E. Herrán (Ed.), Claves de la educación Pikler-Lóczy: Compilación de 20 artículos escritos por sus creadoras (pp. 89-114). Asociación Pikler-Lóczy de Hungría. 
Falk, J. (2018b). Claves de la continuidad en la educación de los niños que viven en una casa cuna. Adaptación, continuidad y salida de la institución. En E. Herrán (Ed.), Claves de la educación Pikler-Lóczy: Compilación de 20 artículos escritos por sus creadoras (pp. 277-344). Asociación Pikler-Lóczy de Hungría.

Falk, J. y Vincze, M. (2018). El desarrollo del control de esfínteres y el interés del niño pequeño hacia las funciones corporales. En E. Herrán (Ed.), Claves de la educación Pikler-Lóczy: Compilación de 20 artículos escritos por sus creadoras (pp. 163-178). Asociación Pikler-Lóczy de Hungría.

Field, F. (2010): The foundation years: preventing poor children becoming poor adults. The report of the independent review on poverty and life chances. Cabinet Office.

Gadeyne, E., Ghesquiere, P. y Onghena, P. (2004). Longitudinal relations between parenting and child adjustment in young children. Journal of Clinical Child and Adolescent Psychology, 33(2), 347-358. https://doi.org/10.1207/s15374424jccp3302_16

González-Mena, J. (2004). What can an orphanage teach us? Lessons from Budapest. Young Children, 59(5), 26-29.

González-Peiteado, M. y Pino-Yuste, M. (2014). Aproximación a las representaciones y creencias del alumnado de magisterio sobre los estilos de enseñanza. Educación XX1, 17(1), 83-110.

Goodman, J. (1988). Constructing a practical philosophy of teaching: A study of preservice teachers' professional perspectives. Teaching and Teacher Education, 4(2), 121-137. https://doi.org/10.1016/0742-051X(88)90013-3

Goodnow, J. J. (1985). Change and variation in ideas about childhood and parenting. En I. E. Sigel, (Ed.), Parental belief systems: The psychological consequences for children (pp. 235-270). Erlbaum.

Gopnik, A. (2010). How babies think. Scientific American, 76-81.

Hamburg, M.E., Finkenauer, C. y Schuengel, C. (2014). Food for love: The role of food offering in empathic emotion regulation. Frontiers in Psychology, 5. https://doi.org/10.3389/fpsyg.2014.00032

Herrán, E. (2013). La educación Pikler-Lóczy. Cuando educar empieza por cuidar. Revista Latinoamericana de Educación Infantil, 2(3), 37-56.

Herrán, E., Orejudo, S., Martínez de Morentín, J. I. y Ordeñana, B. (2014). Actitudes docentes y autonomía en Educación Infantil 0-2: Un estudio exploratorio en la Comunidad Autónoma del País Vasco (CAPV). Revista de Educación, 365, 150-176.

Hoffman, M. L. (1970). Conscience, personality and socialization techniques. Human Development, 13, 90-126. https://doi.org/10.1159/000270884

Kamii, K. (1982). La autonomía como objetivo de la educación: implicaciones de la teoría de Piaget. Infancia y Aprendizaje, 18, 3-32. https://doi.org/10.1080/02103702.1982.10821934

Kellerhalls, J. y Montandon, C. (1997). Les styles éducatifs. En F. De Singly (Dir.), La famille l'état des savoirs (pp. 194-200). Éditions La Découverte.

Loizou, E. y Recchia, S. (2018). In-Service Infant Teachers Re-Envision Their Practice Through a Professional Development Program. Early Education and Development, 29(1), 91-103. https://doi.org/10.1080/10409289.2017.1343561

Lortie, D. C. (2002). Schoolteacher: A sociological study. University of Chicago Press.

Maccoby, E. E. y Martin, J. A. (1983). Socialization in the context of the family: parent-child interaction. En E. M., Hetherington \& P. H., Mussen (Eds.), Handbook of child psychology: vol. 4. Socialization, personality and social development (pp. 1-101). Wiley. 
Meirink, J., Meijer, P., Verloop, N. y Bergen, T. (2009). Understanding teacher learning in secondary education: The relations of teacher activities to changed beliefs about teaching and learning. Teaching and Teacher Education, 25(1), 89-100.

https://doi.org/10.1016/j.tate.2008.07.003.

Moffitt, T. E., Arseneault, L., Belsky, D., Dickson, N., Hancox, R. J., Harrington, H. L., Houts, R., Poulton, R., Roberts, B., Ross, S., Sears, M., Thomson, M. y Caspi, A. (2011). A gradient of childhood self-control predicts health, wealth, and public safety. PNAS, 108, 2693-2698. https://doi.org/10.1073/pnas.1010076108

Morales, P. (2000). Medición de actitudes en psicología y educación. Universidad Pontificia Comillas.

Opfer, V. D. y Pedder, D. (2011). Conceptualizing teacher professional learning. Review of Educational Research, 81(3), 376-407. https://doi.org/10.3102/0034654311413609

Opfer, V. D., Pedder, D. G. y Lavicza, Z. (2011). The role of teachers' orientation to learning in professional development and change: a national study of teachers in England. Teaching and Teacher Education, 27(2), 443-53. https://doi.org/10.1016/j.tate.2010.09.014

Pikler, E. (2018). La competencia del bebé. En E. Herrán (Ed.), Claves de la educación Pikler-Lóczy: Compilación de 20 artículos escritos por sus creadoras (pp. 59-72). Asociación Pikler-Lóczy de Hungría.

Pontes, A., Poyato, F. J. y Oliva, J. M. (2016). Concepciones sobre evaluación en la formación inicial del profesorado de ciencias, tecnología y matemáticas. Revista Iberoamericana de Evaluación Educativa, 9(1), 91-107. https://doi.org/10.15366/riee2016.9.1.006

Poulton, R., Moffitt, T. E. y Silva, P. A. (2015). The Dunedin Multidisciplinary Health and Development Study: overview of the first 40 years, with an eye to the future. Social Psychiatry and Psychiatric Epidemiology, 50(5), 679-693. https://doi.org/10.1007/s00127-015-1048-8

Raby, K. L., Lawler, J. M., Shlafer, R. J., Hesemeyer, P. S., Collins, W. A. y Sroufe, L. A. (2015). The interpersonal antecedents of supportive parenting: a prospective, longitudinal study from infancy to adulthood. Developmental Psychology, 51(1), 115-123. https://doi.org/10.1037/a0038336

Raths, J. (2001). Teachers' beliefs and teaching beliefs. Early Childhood Research \& Practice, 3(1). Recuperado de https://bit.ly/2DXyLB3

Ribeiro, D. y Flores, M. A. (2016). Conceptions and Practices of Assessment in Higher Education: A Study of Portuguese University Teachers. Revista Iberoamericana de Evaluación Educativa, 9(1), 9-29. https://doi.org/10.15366/riee2016.9.1.001

Rollins, B. C. y Thomas, D. L. (1979). Parental support, power and control techniques in the socialization of children. En E. R. Burr et al. (Eds.), Contemporary theories about the family (pp. 317-364). Free Press.

Samuelowicz, K. y Bain, J. D. (2002) Identifying academics' orientations to assessment practice. Higher Education, 43(2), 173-201. https://doi.org/10.1023/A:1013796916022

Sánchez-Rodríguez, J. (2014). La intervención desde la psicomotricidad relacional en la psicosis infantil. Revista Iberoamericana de Psicomotricidad y Técnicas Corporales, 39, 26-40.

Solís, M. (2015). The dilemma of combining positive and negative items in scales. Psicothema, 27(2), 192-199. https://doi.org/10.7334/psicothema2014.266

Schwartz, S. (2003). A proposal for measuring value orientations across nations. En Qestionnaire development report of the european social survey. Recuperado de https://bit.ly/2xq7SAg 
Tardos, A. y Vasseur-Paumelle A. (2018). Reglas y límites en la guardería, adquisición de actitudes. En E. Herrán (Ed.), Claves de la educación Pikler-Lóczy: Compilación de 20 artículos escritos por sus creadoras (pp. 377-392). Asociación Pikler-Lóczy de Hungría.

Tarullo, A., Obradovic, J. y Gunnar, M. (2009). Self-control and the developing brain. Zero to tree. January, 31-37.

Thompson, A. G. (1992). Teachers' beliefs and conceptions: A synthesis of the research. En D. Grouws (Ed.), Handbook of research on mathematics teaching and learning (pp. 127-146). Macmillan.

Tomasello, M. (2007). Los orígenes culturales de la cognición humana. Amorrortu.

Tonyan, H. A., Mamikonian-Zarpas, A. y Chien, D. (2013). Do they practice what they preach? An Ecocultural, multidimensional, group-based examination of the relationship between beliefs and behaviours among child care providers. Early Child Development and Care, 183(12), 18531877. https://doi.org/10.1080/03004430.2012.759949

Trigwell, K. y Prosser, M. (1996). Changing approaches to teaching: A relational perspective. Studies in Higher Education, 21(3), 275-284. https://doi.org/10.1080/03075079612331381211

Vartuli, S. y Rohs, J. (2009). Early childhood prospective teacher pedagogical belief shifts over time. Journal of Early Childhood Teacher Education, 30(4), 310-327. https://doi.org/10.1080/10901020903320262

Vincze, M. (2018). La comida del bebé: del biberón a la autonomía En E. Herrán (Ed.), Claves de la educación Pikler-Lóczy: Compilación de 20 artículos escritos por sus creadoras (pp. 207-232). Asociación Pikler-Lóczy de Hungría.

Wallon, H. (1985). La vida mental. Crítica.

\section{Breve Cv de los autores}

\section{Elena Herrán Izagirre}

Profesora agregada de la Universidad del País Vasco del departamento de Psicología Evolutiva y de la Educación. Investiga en desarrollo temprano, en intervención educativa en Educación Infantil y en formación del profesorado de esta etapa. En estos ámbitos es autora de varios artículos, ha impartido lecciones y presentado comunicaciones y ponencias en cursos, congresos y jornadas de formación para profesionales de la educación infantil. ORCID ID: https://orcid.org/0000-0001-8700-6103. Email: elena.herran@ehu.eus

\section{Nuria Galende Pérez}

Profesora agregada (contratada doctora) de la Universidad del País Vasco. Trabaja en la Facultad de Educación de Bilbao, en el departamento de Psicología Evolutiva y de la Educación. Imparte docencia e investiga principalmente en áreas relacionadas con el desarrollo temprano, intervención educativa en Educación Infantil y Primaria, y en formación del profesorado en ambas etapas. En estos ámbitos, es autora de varios artículos y ha participado en diversos congresos nacionales e internacionales. ORCID ID: https://orcid.org/0000-0002-2 195-6912. Email: nuria.galende@ehu.eus 


\section{Gorka Etxebarria Elordui}

Profesor laboral interino de Universidad en la Universidad del País Vasco, en concreto en la Facultad de Educación de Bilbao. Pertenece al departamento de Didáctica y Organización escolar e investiga en desarrollo temprano. Actualmente es doctorando en el programa de Psicodidáctica (Psicología de la Educación y Didácticas Específicas) y ha participado en varios congresos de carácter internacional. ORCID ID: https://orcid.org/o000-0001-6726-5490. Email: gorka.etxebarriae@ehu.eus 